\title{
Two-Dimensional Direction-of-Arrivals Estimation Based on One-Dimensional Search Using Rank Deficiency Principle
}

\author{
Feng-Gang Yan, ${ }^{1}$ Zhi-Kun Chen, ${ }^{2}$ Ming-Jian Sun, ${ }^{1}$ Yi Shen, ${ }^{2}$ and Ming Jin ${ }^{1}$ \\ ${ }^{1}$ Harbin Institute of Technology at Weihai, Weihai 264209, China \\ ${ }^{2}$ Harbin Institute of Technology, Harbin 150001, China \\ Correspondence should be addressed to Ming-Jian Sun; sunmingjian@hit.edu.cn
}

Received 21 September 2015; Accepted 14 December 2015

Academic Editor: Andy W. H. Khong

Copyright (C) 2015 Feng-Gang Yan et al. This is an open access article distributed under the Creative Commons Attribution License, which permits unrestricted use, distribution, and reproduction in any medium, provided the original work is properly cited.

\begin{abstract}
A novel efficient method for two-dimensional (2D) direction-of-arrivals (DOAs) estimation is proposed to reduce the computational complexity of conventional 2D multiple signal classification (2D-MUSIC) algorithm with uniform rectangular arrays (URAs). By introducing two electrical DOAs, the formula of 2D-MUSIC is transformed into a new one-dimensional (1D) quadratic optimal problem. This $1 \mathrm{D}$ quadratic optimal problem is further proved equivalent to finding the conditions of noise subspace rank deficiency (NSRD), which can be solved by an efficient 1D spectral search, leading to a novel NSRD-MUSIC estimator accordingly. Unlike 2D-MUSIC with exhaustive 2D search, the proposed technique requires only an efficient $1 \mathrm{D}$ one. Compared with the estimation of signal parameter via rotation invariance techniques (ESPRIT), NSRD-MUSIC has a significantly improved accuracy. Moreover, the new algorithm requires no pair matching. Numerical simulations are conducted to verify the efficiency of the new estimator.
\end{abstract}

\section{Introduction}

Estimation of direction-of-arrival (DOA) of multiple narrowband signals using sensor arrays is of great interest in many fields such as radar, sonar, and wireless communications $[1,2]$. Over several decades, this topic has been extensively addressed and numerical algorithms have been developed. Among those approaches, the multiple signal classification (MUSIC) [3] algorithm firstly exploits the orthogonality between the signal and the noise subspaces to achieve a so-called superresolution DOA estimate. Because those two subspaces are perfectly orthogonal to each other when the signal-to-noise ratio (SNR) is sufficiently high, the MUSIC algorithm can resolve two sources as closely spaced as possible in theory. Another outstanding advantage of the MUSIC algorithm over the other superresolution methods is that the former can be used with arbitrary array geometries [4]. However, the computational complexity of MUSIC is prohibitively expensive for real-time applications, owing to an involved matrix decomposition step for the signal or/and the noise subspace estimation and a tremendous spectral search step for final DOA estimates [5].
In practical applications, it is two-dimensional (2D) DOAs (2D DOAs) (i.e., elevation and azimuth angles) that people are usually most concerned about. Estimators such as MUSIC and estimation of signal parameter via rotation invariance technique (ESPRIT) [6] have been studied in onedimensional (1D) situations and further extended to 2D scenarios [7]. Due to the increase in the dimensionality of the 2D DOA estimation problem, the complexity of DOA estimation process is severely affected by the array geometry [8], and further the pair matching (i.e., association or alignment) of the estimated elevation and azimuth angles is usually required [9]. Since, for arbitrary array configurations, the elevation and azimuth angles are extremely coupled with each other in the array response manifold, the heavy 2D spectral search cannot be avoided. To reduce the complexity, researchers are obliged to focus on reducing the computational burden of the 2D DOA estimation problem with various specified array structures.

Planar sensor arrays composed of two or more uniform linear arrays (ULAs) with simple geometry configurations have received considerable attention. Exploiting specified geometries such as L-shaped [10, 11], Y-shaped [12], and 
Z-shaped arrays [13], 2D DOAs of multiple incident signals can be estimated with reduced computational burden by applying most $1 \mathrm{D}$ subspace-based estimation methods including MUSIC and ESPRIT. Special array configurations allow utilizing the shift invariance and the partition of array response vector of two overlapping subarrays of each ULA such that the signal or/and noise subspaces can be estimated without eigenvalue decomposition (EVD) or singular valued decomposition (SVD). Nevertheless, spectral search is still required for those methods, and, as a matter of fact, the complexity of spectral search is substantially heavier than that of subspace decomposition, especially for 2D DOAs estimates [14]. Moreover, because the total arrays are usually divided into several subarrays in those methods, the array aperture is in fact reduced and DOA estimation accuracy may be sacrificed [15].

Uniform rectangular array (URA) is another frequently discussed array structure. A geometric formulation of the ESPRIT algorithm exploiting subarrays of URA is presented to reparameterize the weighted subspace fitting (WSF) algorithm in [16], leading naturally to an extension to the $2 \mathrm{D}$ DOA estimation problem. With high dimensional signal processing, the 2D subspace-based algorithms in [17] can provide precise estimates at the expense of high computational complexity exponentially increasing with the size of the rectangular arrays. To reduce the computational load, a tree structure algorithm by first performing 1D spatial smoothing and then 1D MUSIC several times successively is proposed in [18] to estimate the azimuth and elevation angles independently. Despite the computational efficiency, almost all of the aforementioned techniques realize 2D DOA estimates at the cost of a reduction in array aperture.

Recently, an outstanding reduced-dimensional MUSIC (RD-MUSIC) algorithm is proposed to avoid the high computational cost within 2D-MUSIC for direction of departure (DOD) and DOA estimation in multiinput multioutput (MIMO) radar [19]. Using DOA information embedded in the velocity sensors, RD-MUSIC starts 1D MUSIC searches for the DOD and DOA in succession without parameter pairing nor $2 \mathrm{D}$ search. In fact, the formula of RD-MUSIC is somewhat similar to that of 2D DOA estimates except that the noise projection matrix in the former is of reduced order and is invertible while that of the latter is not. Following this idea, we present in this paper a new efficient technique for 2D DOAs estimation with URAs. With two introduced electrical angles, we show that the formula of 2D-MUSIC can be transformed into a new $1 \mathrm{D}$ quadratic optimal problem. Further analysis demonstrates that this 1D quadratic optimal problem is equivalent to finding the conditions of noise subspace rank deficiency (NSRD), which can be solved by an efficient 1D spectral search. Consequently, a novel NSRDMUSIC estimator at hand is derived. Unlike 2D-MUSIC with exhaustive $2 \mathrm{D}$ search, the proposed technique requires only an efficient 1D one. Compared with ESPRIT, NSRD-MUSIC has a significantly improved Root Mean Square Error (RMSE) performance.

The outline of this paper is as follows. The narrow-band signal model and the conventional 2D-MUSIC algorithm are introduced in Section 2. Two transformed electrical angles

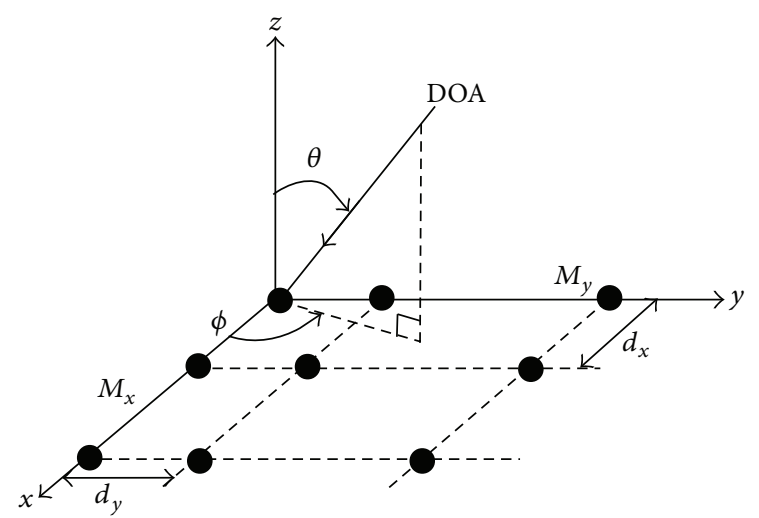

FIGURE 1: Array geometry of the URA.

reformulating the 2D-MUSIC algorithm into a new $1 D$ optimum problem is then considered, and the proposed method is addressed in detail in Section 3. The complexity of our method is analyzed in Section 4 and simulation results are conducted and discussed to validate the effectiveness of new method in Section 5.

\section{Signal Model and Standard 2D-MUSIC}

Assume that there are $K$ narrow-band signals with unknown $2 \mathrm{D}$ DOAs $(\boldsymbol{\theta}, \boldsymbol{\phi}) \triangleq\left\{\left(\theta_{1}, \phi_{1}\right),\left(\theta_{2}, \phi_{2}\right), \ldots,\left(\theta_{K}, \phi_{K}\right)\right\}$ simultaneously incident on URA of $M=M_{x} M_{y}$ sensors, where $M_{x}$ and $M_{y}$ are the numbers of elements in $x$-direction and $y$ direction, respectively. The corresponding element spacing symbols are $d_{x}$ and $d_{y}$, as depicted in Figure 1 . The azimuth angle $\theta$ is defined as the one between the wave direction and the $z$-axis while the elevation angle $\phi$ is defined as the one between the $x$-axis and the projection of wave direction onto the $x$-y plane. Note that $\theta \in[0, \pi / 2]$ and $\phi \in[0,2 \pi]$. It is assumed that there are $N$ snapshots available; $M \times 1$ array output vector at snapshot $t$ is given by

$$
\mathbf{x}(t)=\mathbf{A s}(t)+\mathbf{n}(t), \quad t=1, \ldots, N,
$$

where

$$
\mathbf{A}=\left[\mathbf{a}\left(\theta_{1}, \phi_{1}\right), \mathbf{a}\left(\theta_{2}, \phi_{2}\right), \ldots, \mathbf{a}\left(\theta_{K}, \phi_{K}\right)\right]
$$

is the $M \times K$ matrix of the signal direction vectors and

$$
\mathbf{a}(\theta, \phi)=\left[\begin{array}{c}
1 \\
e^{j\left(2 \pi / \kappa_{c}\right)\left(d_{x} \sin \theta \cos \phi+d_{y} \sin \theta \sin \phi\right)} \\
\vdots \\
e^{j\left(2 \pi / \kappa_{c}\right)\left[\left(M_{x}-1\right) d_{x} \sin \theta \cos \phi+\left(M_{y}-1\right) d_{y} \sin \theta \sin \phi\right]}
\end{array}\right]
$$

is the $M \times 1$ steering vector. In addition, $j \triangleq \sqrt{-1}$ and $\mathbf{s}(t)$ is the $K \times 1$ vector of source waveforms; $\mathbf{n}(t)$ is the $M \times 1$ vector of white sensor noise; $\kappa_{c}$ is the center wavelength; $(\cdot)^{T}$ is the matrix transpose.

The estimate of the $M \times M$ array output covariance matrix (AOCM)

$$
\mathbf{R}=E\left[\mathbf{x}(t) \mathbf{x}^{H}(t)\right]=\mathbf{A} \mathbf{R}_{s} \mathbf{A}^{H}+\sigma_{n}^{2} \mathbf{I}_{M}
$$


is given by

$$
\widehat{\mathbf{R}}=\frac{1}{N} \sum_{t=1}^{N} \mathbf{x}(t) \mathbf{x}^{H}(t),
$$

where $\mathbf{R}_{s}=E\left(\mathbf{s}(t) \mathbf{s}^{H}(t)\right)$ is $K \times K$ signal covariance matrix; I is $M \times M$ identity matrix; $\sigma_{n}^{2}$ is the noise variance; $E(\cdot)$ is the mathematical expectation; and $(\cdot)^{H}$ is the matrix Hermitian transpose.

2.1. Subspace Decomposition and 2D-MUSIC Algorithm. The EVDs of the theoretical AOCM (4) and the practical estimated AOCM (5) can be defined in a standard way:

$$
\begin{aligned}
& \mathbf{R}=\mathbf{V}_{s} \boldsymbol{\Pi}_{s} \mathbf{V}_{s}^{H}+\mathbf{V}_{n} \boldsymbol{\Pi}_{n} \mathbf{V}_{n}^{H}, \\
& \widehat{\mathbf{R}}=\widehat{\mathbf{V}}_{s} \widehat{\boldsymbol{\Pi}}_{s} \widehat{\mathbf{V}}_{s}^{H}+\widehat{\mathbf{V}}_{n} \widehat{\boldsymbol{\Pi}}_{n} \widehat{\mathbf{V}}_{n}^{H},
\end{aligned}
$$

where

$$
\begin{aligned}
& \mathbf{V}_{s}=\left[\mathbf{v}_{1}, \ldots, \mathbf{v}_{K}\right], \\
& \mathbf{V}_{n}=\left[\mathbf{v}_{K+1}, \ldots, \mathbf{v}_{M}\right], \\
& \boldsymbol{\Pi}_{s}=\operatorname{diag}\left\{\pi_{1}, \ldots, \pi_{K}\right\}, \\
& \boldsymbol{\Pi}_{n}=\operatorname{diag}\left\{\pi_{K+1}, \ldots, \pi_{M}\right\}, \\
& \widehat{\mathbf{v}}_{s}=\left[\widehat{\mathbf{v}}_{1}, \ldots, \widehat{\mathbf{v}}_{K}\right], \\
& \widehat{\mathbf{V}}_{n}=\left[\widehat{\mathbf{v}}_{K+1}, \ldots, \widehat{\mathbf{v}}_{M}\right], \\
& \widehat{\boldsymbol{\Pi}}_{s}=\operatorname{diag}\left\{\widehat{\pi}_{1}, \ldots, \widehat{\pi}_{K}\right\}, \\
& \widehat{\Pi}_{n}=\operatorname{diag}\left\{\widehat{\pi}_{K+1}, \ldots, \widehat{\pi}_{M}\right\}
\end{aligned}
$$

with the subscripts $s$ and $n$ standing for the signal and noise subspace, respectively.

Based on the orthogonality between $\operatorname{span}\left(\mathbf{V}_{s}\right)$ and $\operatorname{span}\left(\mathbf{V}_{n}\right)$, conventional 2D-MUSIC algorithm [3] suggests searching the following function:

$$
P_{2 \text { D-MUsiC }}(\theta, \phi)=\frac{1}{\mathbf{a}^{H}(\theta, \phi) \widehat{\mathbf{V}}_{n} \widehat{\mathbf{V}}_{n}^{H} \mathbf{a}(\theta, \phi)}
$$

over the total $2 \mathrm{D}$ angular field-of-view $[0, \pi / 2] \times[0,2 \pi]$ with a fine grid. $K$ peaks of $P_{2 \mathrm{D}-\mathrm{MUSIC}}(\theta)$ indicate source DOAs. One of the advantages of the 2D-MUSIC algorithm over other subspace-based methods is its nondependence on array configurations [4]. However, the complexity of this spectral search step is typically substantially high since, for each point, the product $\mathbf{a}^{H}(\theta, \phi) \widehat{\mathbf{V}}_{n} \widehat{\mathbf{V}}_{n}^{H} \mathbf{a}(\theta, \phi)$ has to be computed.

\section{The Proposed Algorithm}

The $2 \mathrm{D}$ steering vector given in (3) represents the nominal array response. Following the notation in [20], $\mathbf{a}\left(\theta_{k}, \phi_{k}\right), k=$ $1, \ldots, K$ can be rewritten as

$$
\mathbf{a}\left(\theta_{k}, \phi_{k}\right)=\mathbf{a}_{x}\left(\psi_{x}\right) \otimes \mathbf{a}_{y}\left(\psi_{y}\right), \quad k=1, \ldots, K,
$$

where $\otimes$ denotes the Kronecker product and the elements of vectors $\mathbf{a}_{x}\left(\psi_{x}\right)$ and $\mathbf{a}_{y}\left(\psi_{y}\right)$ are given by

$$
\begin{aligned}
& {\left[\mathbf{a}_{x}\left(\psi_{x}\right)\right]_{m_{x}}=e^{j\left(2 \pi / \kappa_{c}\right) \psi_{x}\left(m_{x}-1\right)}, \quad m_{x}=1, \ldots, M_{x},} \\
& {\left[\mathbf{a}_{y}\left(\psi_{x}\right)\right]_{m_{y}}=e^{j\left(2 \pi / \kappa_{c}\right) \psi_{y}\left(m_{y}-1\right)}, \quad m_{y}=1, \ldots, M_{y},}
\end{aligned}
$$

where $[\cdot]_{m}$ denote the $m$ th element of the embraced vector. Electrical angles $\psi_{x}$ and $\psi_{y}$ are related to the physical azimuth and elevation angles by

$$
\begin{aligned}
& \psi_{x}=d_{x} \sin \theta \cos \phi \\
& \psi_{y}=d_{y} \sin \theta \sin \phi
\end{aligned}
$$

and the corresponding inverse relations for obtaining azimuth and elevation angles are

$$
\begin{aligned}
& \phi=\tan ^{-1}\left(\frac{d_{y}}{d_{x}} \frac{\psi_{y}}{\psi_{x}}\right), \\
& \theta=\sin ^{-1}\left(\sqrt{\frac{\psi_{x}^{2}}{d_{x}^{2}}+\frac{\psi_{y}^{2}}{d_{y}^{2}}}\right) .
\end{aligned}
$$

Let us define

$$
\begin{aligned}
& \mathbf{P}\left(\psi_{x}, \psi_{y}\right) \\
& \triangleq\left[\mathbf{a}_{x}\left(\psi_{x}\right) \otimes \mathbf{a}_{y}\left(\psi_{y}\right)\right]^{H} \mathbf{V}_{n} \mathbf{V}_{n}^{H}\left[\mathbf{a}_{x}\left(\psi_{x}\right) \otimes \mathbf{a}_{y}\left(\psi_{y}\right)\right] .
\end{aligned}
$$

Then the spectral search involved in the 2D-MUSIC algorithm is equivalent to the following optimization problem:

$$
\begin{array}{cl}
\min _{\left(\psi_{x}, \psi_{y}\right)} & \widehat{\mathbf{P}}\left(\psi_{x}, \psi_{y}\right) \\
\text { s.t. } & \left(\psi_{x}, \psi_{y}\right) \in\left[-d_{x}, d_{x}\right] \times\left[-d_{y}, d_{y}\right] .
\end{array}
$$

Observing that $\mathbf{a}_{x}\left(\psi_{x}\right) \otimes \mathbf{a}_{y}\left(\psi_{y}\right)=\left[\mathbf{a}_{y}\left(\psi_{y}\right) \otimes \mathbf{I}_{M_{x}}\right] \mathbf{a}_{x}\left(\psi_{x}\right),(13)$ can be rewritten as

$$
\begin{aligned}
& \mathbf{P}\left(\psi_{x}, \psi_{y}\right)=\mathbf{a}_{x}^{H}\left(\psi_{x}\right)\left[\mathbf{a}_{y}\left(\psi_{y}\right) \otimes \mathbf{I}_{M_{x}}\right]^{H} \\
& \cdot \mathbf{V}_{n} \mathbf{V}_{n}^{H}\left[\mathbf{a}_{y}\left(\psi_{y}\right) \otimes \mathbf{I}_{M_{x}}\right] \mathbf{a}_{x}\left(\psi_{x}\right) \triangleq \mathbf{a}_{x}^{H}\left(\psi_{x}\right) \\
& \cdot \mathbf{Q}_{\psi_{y}} \mathbf{a}_{x}\left(\psi_{x}\right),
\end{aligned}
$$

where

$$
\mathbf{Q}_{\psi_{y}} \triangleq\left[\mathbf{a}_{y}\left(\psi_{y}\right) \otimes \mathbf{I}_{M_{x}}\right]^{H} \mathbf{V}_{n} \mathbf{V}_{n}^{H}\left[\mathbf{a}_{y}\left(\psi_{y}\right) \otimes \mathbf{I}_{M_{x}}\right] .
$$

Therefore, the optimization problem in (14) can be reconstructed as follows:

$$
\begin{aligned}
\min _{\left(\psi_{x}, \psi_{y}\right)} & \mathbf{a}_{x}^{H}\left(\psi_{x}\right) \widehat{\mathbf{Q}}_{\psi_{y}} \mathbf{a}_{x}\left(\psi_{x}\right) \\
\text { s.t. } & \left(\psi_{x}, \psi_{y}\right) \in\left[-d_{x}, d_{x}\right] \times\left[-d_{y}, d_{y}\right] .
\end{aligned}
$$


To solve the above quadratic optimization, it is suggested in [19] to use the following constraint:

$$
\mathbf{e}_{1}^{H} \mathbf{a}_{x}\left(\psi_{x}\right)=1, \quad \mathbf{e}_{1} \triangleq[1, \underbrace{0, \ldots, 0}_{M_{x}-1 \text { zeros }}]
$$

to eliminate the trivial solution $\mathbf{a}_{x}\left(\psi_{x}\right)=\mathbf{0}$, leading to a so-called RD-MUSIC estimator with the following search function:

$$
P_{\mathrm{RD}-\mathrm{MUSIC}}\left(\psi_{y}\right)=\left[\widehat{\mathbf{Q}}_{\psi_{y}}^{-1}\right]_{(1,1)},
$$

where $[\cdot]_{(1,1)}$ is the $(1,1)$ th element of the embraced matrix. The electrical angle $\psi_{y}^{k}, k=1, \ldots, K$ can be estimated by an efficient one-dimensional spectral search over $\left[-d_{y}, d_{y}\right]$ for $K$ largest peaks of $P_{\mathrm{RD}-\mathrm{MUSIC}}\left(\psi_{y}\right)$. However, the constraint $\mathbf{e}_{1}^{H} \mathbf{a}_{x}\left(\psi_{x}\right)=1$ in fact only warrants that the first element of $\mathbf{a}_{x}\left(\psi_{x}\right)$ equals one. We now propose a new NSRD-MUSIC method which has a similar computational complexity as compared to the RD-MUSIC algorithm.

Because $\mathbf{a}_{x}\left(\psi_{x}\right) \neq \mathbf{0}$, it is clear that

$$
\mathbf{a}_{x}^{H}\left(\psi_{x}\right) \mathbf{Q}_{\psi_{y}} \mathbf{a}_{x}\left(\psi_{x}\right)=0
$$

holds if and only if $M_{x} \times M_{x}$ matrix $\mathbf{Q}_{\psi_{y}}$ is of rank deficiency; that is,

$$
\mathbf{a}_{x}^{H}\left(\psi_{x}\right) \mathbf{Q}_{\psi_{y}} \mathbf{a}_{x}\left(\psi_{x}\right)=0 \Longleftrightarrow \operatorname{rank}\left(\mathbf{Q}_{\psi_{y}}\right)<M_{x} .
$$

Noting that [21]

$$
\begin{aligned}
\operatorname{rank}\left[\mathbf{a}_{y}\left(\psi_{y}\right) \otimes \mathbf{I}_{M_{x}}\right] & =\operatorname{rank}\left[\mathbf{a}_{y}\left(\psi_{y}\right)\right] \cdot \operatorname{rank}\left(\mathbf{I}_{M_{x}}\right) \\
& =M_{x}, \\
\operatorname{rank}\left(\mathbf{V}_{n} \mathbf{V}_{n}^{H}\right) & =\operatorname{rank}\left(\mathbf{V}_{n}\right)=M-K,
\end{aligned}
$$

for $M-K \geq M_{x}$ or equivalently for

$$
K \leq M-M_{x}
$$

$\mathbf{Q}_{\psi_{y}}$ is a rank-deficient matrix if and only if $\psi_{y}$ is the electrical angle of source DOAs. Therefore, we have

$$
\begin{aligned}
\operatorname{rank}\left(\mathbf{Q}_{\psi_{y}}\right)<M_{x} \Longleftrightarrow \psi_{y}^{k}=d_{y} \sin \theta_{k} \sin \phi_{k}, & \\
& k=1, \ldots, K .
\end{aligned}
$$

Based on the above analysis, $K$ electrical angle that related the source DOAs, that is, $\psi_{y}^{k}, k=1, \ldots, K$, can be estimated by searching

$$
P_{\text {NSRD-MUSIC }}\left(\psi_{y}\right)=\frac{1}{\operatorname{Eig}_{\min }\left[\widehat{\mathbf{Q}}_{\psi_{y}}\right]}
$$

or equivalently by searching

$$
P_{\text {NSRD-MUSIC }}\left(\psi_{y}\right)=\frac{1}{\operatorname{Det}\left[\widehat{\mathbf{Q}}_{\psi_{y}}\right]}
$$

over $\left[-d_{y}, d_{y}\right]$ for $K$ largest peaks of $P_{\text {NSRD-MUSIC }}\left(\psi_{y}\right)$. Here, Eig $_{\min }[\cdot]$ and Det $[\cdot]$ stand for the minimum eigenvalue and the determinant of the embraced matrix, respectively. $K$ angles relating to $K$ largest peaks of $P_{\text {NSRD-MUSIC }}\left(\psi_{y}\right)$ should be the electrical angles $\psi_{y}^{k}, k=1, \ldots, K$.

With $\psi_{y}^{k}, k=1, \ldots, K$, we can compute $K$ steering vectors corresponding to $\psi_{x}^{k}, k=1, \ldots, K$ as follows [19]:

$$
\mathbf{a}_{x}\left(\psi_{x}^{k}\right)=\frac{\mathbf{Q}_{\psi_{y}^{k}}^{-1} \mathbf{e}_{1}}{\mathbf{e}_{1}^{H} \mathbf{Q}_{\psi_{y}^{k}}^{-1} \mathbf{e}_{1}}, \quad k=1, \ldots, K .
$$

Combing (10) and (27) leads to

$$
\mathbf{g}_{k} \triangleq-L\left[\mathbf{a}_{x}\left(\psi_{x}^{k}\right)\right]=\left[\begin{array}{c}
0 \\
1 \\
2 \\
\vdots \\
M_{x}-1
\end{array}\right] \begin{array}{ll} 
\\
\\
& k=1, \ldots, K,
\end{array}
$$

where $\angle(\cdot)$ stands for phase angles for each element of the embrace vector. We now exploit the least squares (LS) principle to estimate $\widehat{\psi}_{x}^{k}, k=1, \ldots, K$. Define

$$
\begin{gathered}
\mathbf{P} \triangleq\left[\begin{array}{cc}
1 & 0 \\
1 & 1 \\
1 & 2 \\
1 & \vdots \\
1 & M_{x}-1
\end{array}\right], \\
\boldsymbol{\xi}_{k} \triangleq\left[\begin{array}{c}
0 \\
\frac{2 \pi}{\kappa_{c}} \psi_{x}^{k}
\end{array}\right] .
\end{gathered}
$$

Then we have

$$
\mathbf{P} \boldsymbol{\xi}_{k}=\mathbf{g}_{k}, \quad k=1, \ldots, K
$$

The LS solution for estimating $\widehat{\boldsymbol{\xi}}_{k}$ with $\widehat{\mathbf{g}}_{k}$ is given by

$$
\widehat{\boldsymbol{\xi}}_{k}=\left(\mathbf{P}^{T} \mathbf{P}\right)^{-1} \mathbf{P}^{T} \widehat{\mathbf{g}}_{k}, \quad k=1, \ldots, K .
$$

Using (29) and (31), $\psi_{x}^{k}, k=1, \ldots, K$ is estimated by

$$
\widehat{\psi}_{x}^{k}=\frac{\kappa_{c}}{2 \pi}\left[\widehat{\boldsymbol{\xi}}_{k}\right]_{2}, \quad k=1, \ldots, K
$$

Now, we have estimated $K$ electrical angles $\psi_{y}^{k}, k=$ $1, \ldots, K$ and $\psi_{x}^{k}, k=1, \ldots, K$. Since, for each $\psi_{y}^{k}, k \in$ $[1, K]$, we can compute a corresponding $\psi_{x}^{k}, k \in[1, K]$ by using (27)-(32), no further pair matching is required for the proposed algorithm. Hence, we can directly insert $\left(\widehat{\psi}_{x}^{k}, \widehat{\psi}_{y}^{k}\right), k=1, \ldots, K$, into (12) to finally obtain the estimates of the azimuth and elevation angles $\left(\widehat{\theta}_{k}, \widehat{\phi}_{k}\right), k=1, \ldots, K$. 


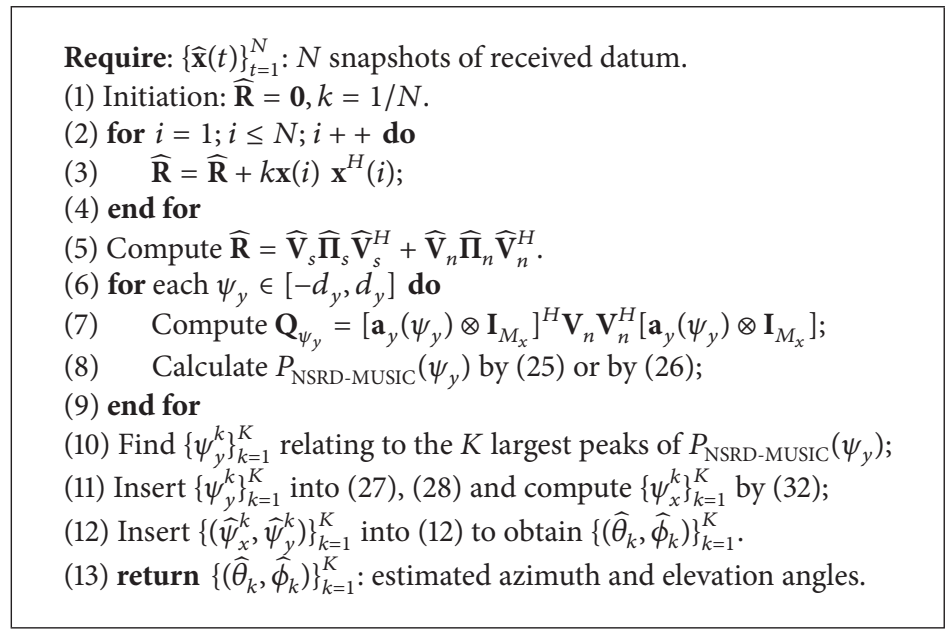

Algorithm 1: The proposed NSRD-MUSIC algorithm.

TABLE 1: Complexity in terms of real-valued operations.

\begin{tabular}{lc}
\hline Algorithms & Primary computations \\
\hline 2D-MUSIC & $\mathcal{O}\left(M^{2} K\right)+\mathcal{O}\left(J_{\theta} \cdot J_{\phi}(M+1)(M-K)\right)$ \\
RD-MUSIC [19] & $\mathcal{O}\left(M^{2} K\right)+\mathcal{O}\left(J_{\psi_{y}} M_{x}^{2} K\right)$ \\
NSRD-MUSIC & $\mathcal{O}\left(M^{2} K\right)+\mathcal{O}\left(J_{\psi_{y}} M_{x}^{2} K\right)$ \\
\hline
\end{tabular}

\section{Complexity Analysis and Summary}

Comparison of primary complexity among three algorithms including 2D-MUSIC [3], RD-MUSIC [19], and the proposed NSRD-MUSIC is shown in Table 1 , where $J_{\theta}, J_{\phi}$, and $J_{\psi_{y}}$ stand for the search numbers along the directions of $\theta, \phi$, and $\psi_{y}$, respectively. The common term $\mathcal{O}\left(M^{2} K\right)$ gives the complexity of EVD on $\mathbf{R}$ [21]. Because 2D-MUSIC involves a 2D spectral search step, it has to compute $J_{\theta} \cdot J_{\phi}$ spectral points. Since RD-MUSIC and the proposed method involve a 1D search step, the two techniques only need to compute $J_{\psi_{y}}$ spectral points. Generally, we have $J_{\theta} \approx J_{\phi} \approx J_{\psi_{y}} \gg M>K$. Therefore, NRSD-MUSIC has a much lower complexity than 2D-MUSIC.

Detailed steps for implementing the proposed NSRDMUSIC algorithm are summarized in Algorithm 1.

\section{Simulation Results}

Simulations with 500 independent Monte Carlo methods have been conducted on UCR composed of $M_{x} \times M_{y}=3 \times 3=$ 9 sensors with half wavelength apart along both $x$ - and $y$ axis to assess the performance of the proposed estimator. It is assumed that there are $K=3$ sources at $\left(\theta_{1}=10^{\circ}, \phi_{1}=20^{\circ}\right)$, $\left(\theta_{2}=30^{\circ}, \phi_{2}=35^{\circ}\right)$, and $\left(\theta_{3}=40^{\circ}, \phi_{3}=150^{\circ}\right)$ that are implying on the array from far-field. The RMSEs for the estimates of source incident angles $\theta$ and $\phi$ are defined as

$$
\mathrm{RMSE}_{\theta} \triangleq \sqrt{\frac{1}{500} \sum_{i=1}^{500}\left(\widehat{\theta}_{i}-\theta\right)^{2}}
$$

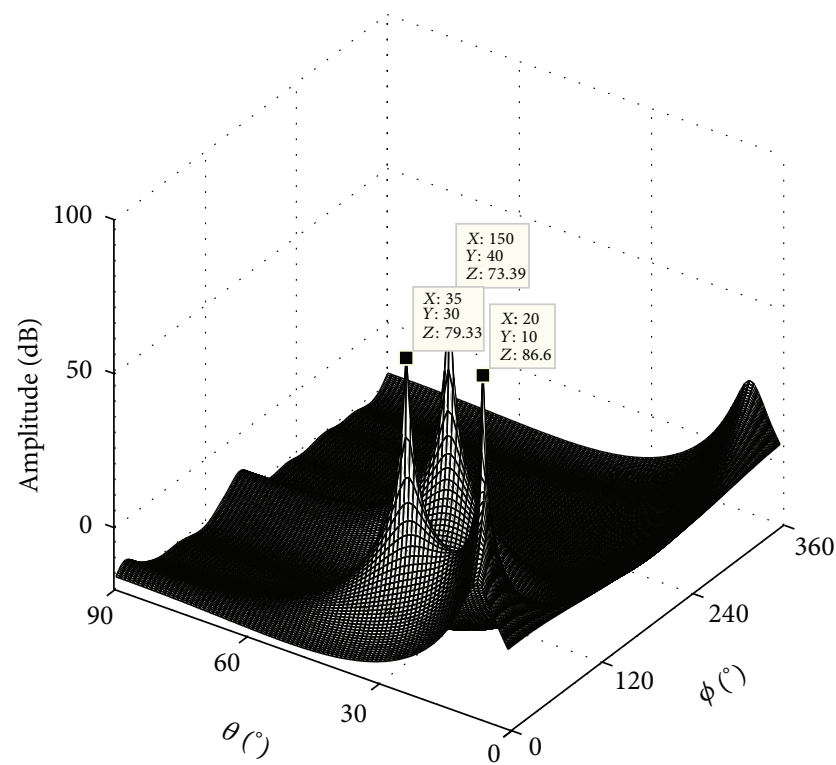

FIgURE 2: Spectrum of 2D-MUSIC.

$$
\mathrm{RMSE}_{\phi} \triangleq \sqrt{\frac{1}{500} \sum_{i=1}^{500}\left(\hat{\phi}_{i}-\phi\right)^{2}}
$$

respectively, where $\theta$ and $\phi$ are the true 2D DOAs while $\widehat{\phi}_{i}$ and $\widehat{\theta}_{i}$ represent the estimated values of the elevation and azimuth angles of the $i$ th trial.

Figures 2 and 3 plot the spectrums of three functions including 2D-MUSIC, RD-MUSIC [19], and the proposed estimator. It is seen from the figures that the proposed method estimates $\psi_{y}$ correctly, which generates corresponding peaks at $\psi_{y}^{1}=\sin \left(\theta_{1}\right) \cos \left(\phi_{1}\right)=\sin \left(10^{\circ}\right) \cos \left(20^{\circ}\right) \approx$ $0.16, \psi_{y}^{2}=\sin \left(\theta_{2}\right) \cos \left(\phi_{2}\right)=\sin \left(30^{\circ}\right) \cos \left(35^{\circ}\right) \approx 0.41$, and $\psi_{y}^{3}=\sin \left(\theta_{3}\right) \cos \left(\phi_{3}\right)=\sin \left(40^{\circ}\right) \cos \left(150^{\circ}\right) \approx-0.56$ as expected. This indicates that $\psi_{y}$ can be estimated by the 


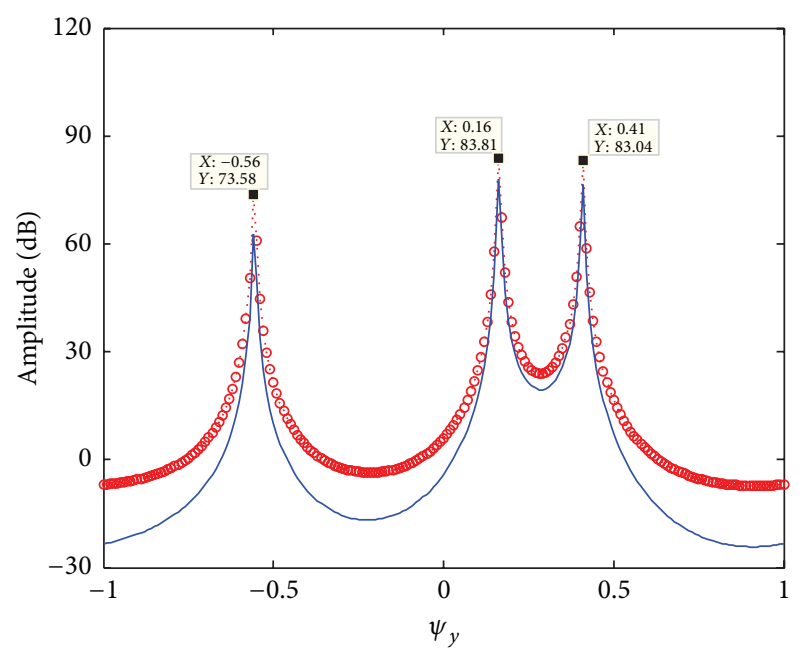

○. RD-MUSIC

- The proposed

FigURE 3: Spectrums of RD-MUSIC and the proposed.

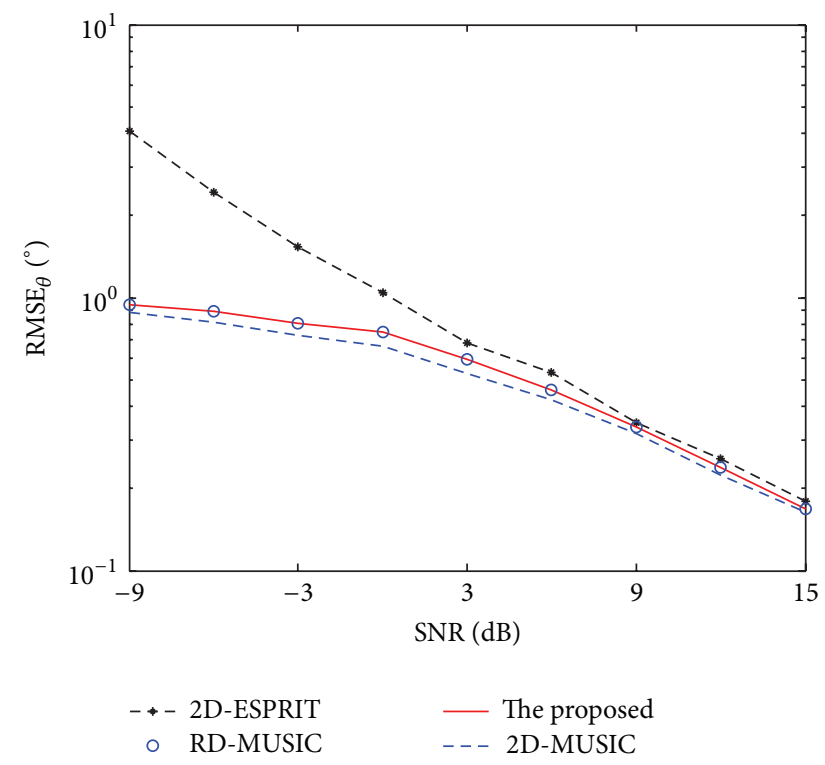

FIGURE 4: RMSE of $\theta$ against the SNR.

proposed method with $1 \mathrm{D}$ spectral search efficiently. Therefore, the proposed method has a much lower computational complexity as compared to 2D-MUSIC.

Figures 4 and 5 compare the estimation RMSEs of $\theta$ by four algorithms including 2D-MUSIC, 2D-ESPRIT, RDMUSIC, and the proposed method. In Figure 4, the results are plotted against the SNR, where the number of snapshots is set as $N=200$. In Figure 5, the results are given as functions of the number of snapshots, where SNR $=10 \mathrm{~dB}$. Note that all the estimation accuracies in both Figures 4 and 5 are respected to the source at $\left(\theta_{2}=30^{\circ}, \phi_{2}=35^{\circ}\right)$. It can be concluded form Figure 4 that the proposed method provides a significantly improved accuracy performance as compared to 2D-ESPRIT over the total range of SNR $=-9 \mathrm{~dB}$

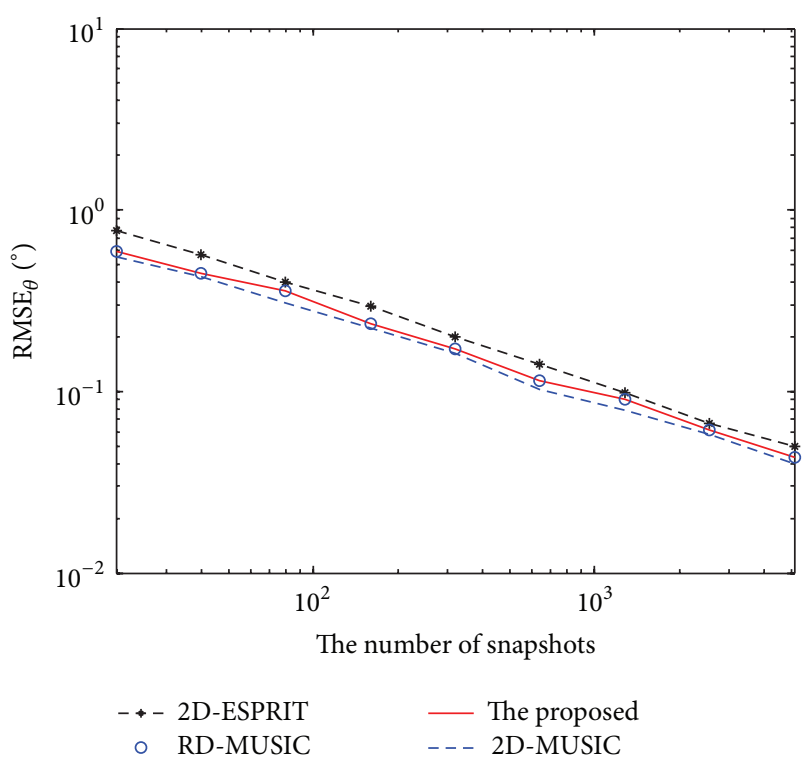

Figure 5: RMSE of $\theta$ against the number of snapshots.

to $\mathrm{SNR}=15 \mathrm{~dB}$. This is because the array aperture is in fact reduced in 2D-ESPRIT while that is not in the proposed method. It can be also concluded form Figure 4 that our method has a very close accuracy to RD-MUSIC and 2DMUSIC, especially for SNR > $3 \mathrm{~dB}$. On the other hand, we can see clearly from Figure 4 that the proposed estimator provides an asymptotically similar accuracy performance to RD-MUSIC and 2D-MUSIC, which is much better than 2DESPRIT.

To further compare the performance of the new method with performances of 2D-MUSIC, 2D-ESPRIT, and RDMUSIC, Figures 6 and 7 give the estimation RMSEs of $\phi$ by different algorithms. The results in Figure 4 are plotted against the SNR while those in Figure 5 are plotted as functions of the number of snapshots. In Figure 4, the number of snapshots is set as $N=200$ and in Figure 5 it is fixed as $\mathrm{SNR}=10 \mathrm{~dB}$. Similarly, all the results in Figures 6 and 7 are respected to the source at $\left(\theta_{2}=30^{\circ}, \phi_{2}=35^{\circ}\right)$. It can be concluded again form Figures 6 and 7 that our method significantly outperforms 2D-ESPRIT, which provides an asymptotically similar accuracy performance to 2D-MUSIC and RD-MUSIC.

Finally, we evaluate the performance of the proposed method against the correlation between the incident signals. We consider the case of three signals $s_{1}(t), s_{2}(t)$, and $s_{3}(t)$, where $s_{1}(t)$ and $s_{2}(t)$ are correlated with each other, and the correlation coefficient is defined as [22]

$$
\rho \triangleq \frac{E\left[s_{1}(t) s_{2}^{*}(t)\right]}{\sqrt{E\left\{\left[s_{1}(t)-m_{s_{1}}(t)\right]^{2}\right\} E\left\{\left[s_{2}(t)-m_{s_{2}}(t)\right]^{2}\right\}}}
$$

where $m_{s_{1}}(t) \triangleq E\left[s_{1}(t)\right]$ and $m_{s_{2}}(t) \triangleq E\left[s_{2}(t)\right]$. The 2D DOAs of the three signals are set as $\left(\theta_{1}=10^{\circ}, \phi_{1}=20^{\circ}\right),\left(\theta_{2}=\right.$ $\left.30^{\circ}, \phi_{2}=35^{\circ}\right)$, and $\left(\theta_{3}=40^{\circ}, \phi_{3}=150^{\circ}\right)$. The RMSEs of DOA estimation versus source correlation with respect to the 


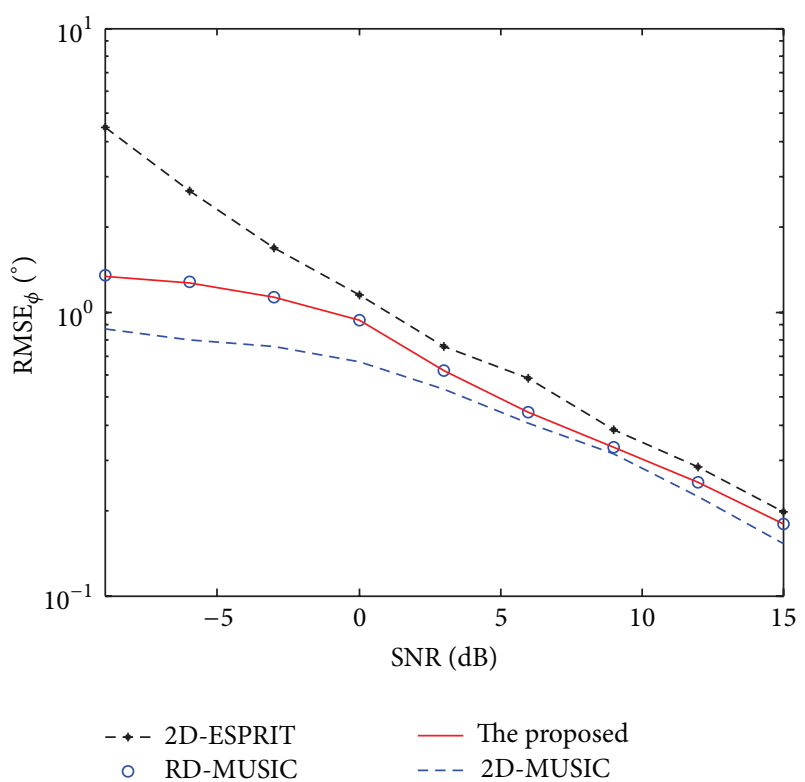

Figure 6: RMSE of $\phi$ against the SNR.

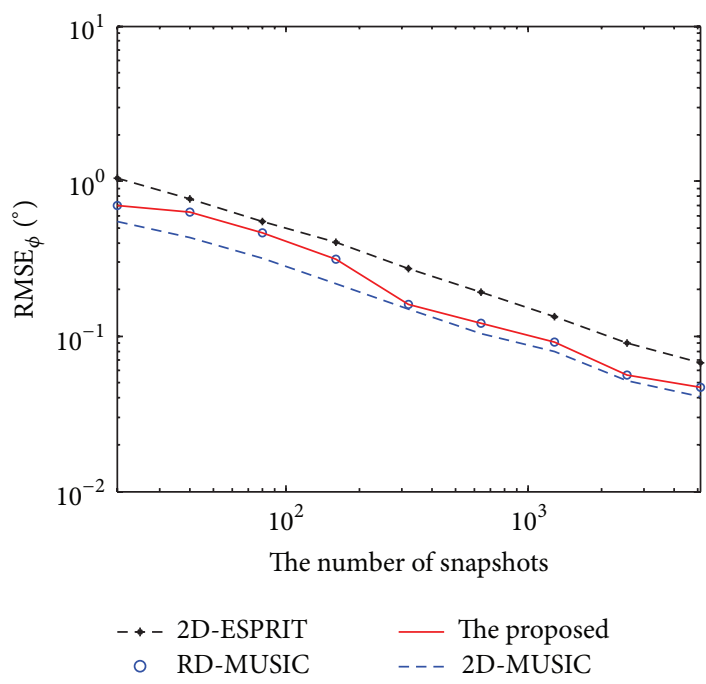

FIGURE 7: RMSE of $\phi$ against the number of snapshots.

second signal are shown in Figures 8 and 9, where the number of snapshots is $N=100, M=9$, and SNR $=20 \mathrm{~dB}$.

It can be seen clearly form Figures 8 and 9 that the accuracies of the RMSEs of all the three algorithms increase as $\rho$ increases. This implies that the accuracies of all the three algorithms decrease when correlated source exists. However, the proposed method has a similar performance to the RDMUSIC algorithm, and it performs closely to the standard 2D-MUSIC over the wide range $\rho=0$ to $\rho=1$. Although the RMSEs of the new method are a little bigger than those of 2D-MUSIC, the complexity of the proposed method is much lower than that of 2D-MUSIC. Therefore, the new estimator makes an efficient trade-off between estimation accuracy and computational complexity.

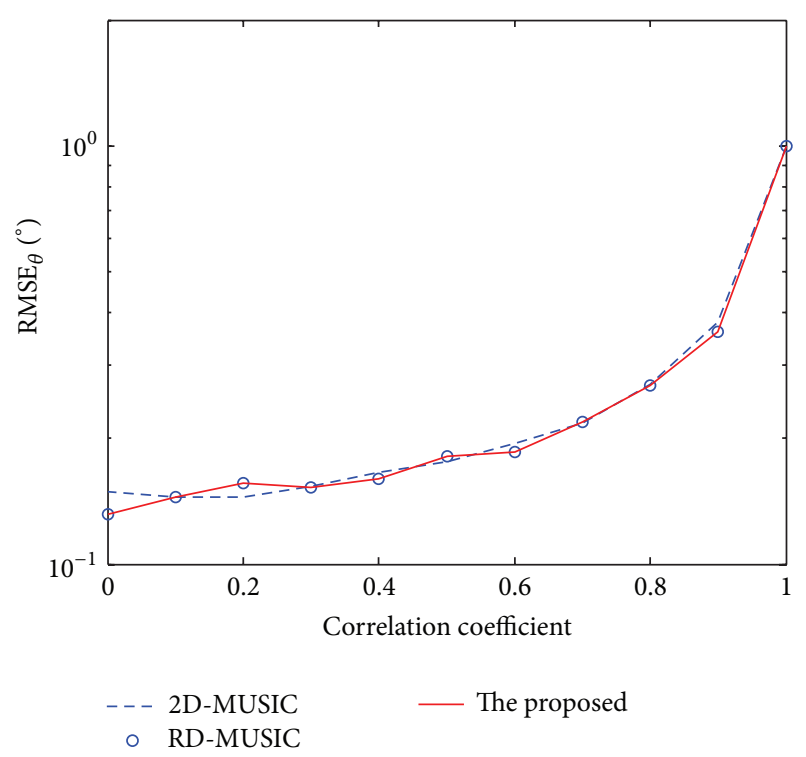

FIGURE 8: RMSE of $\theta$ against correlation coefficient.

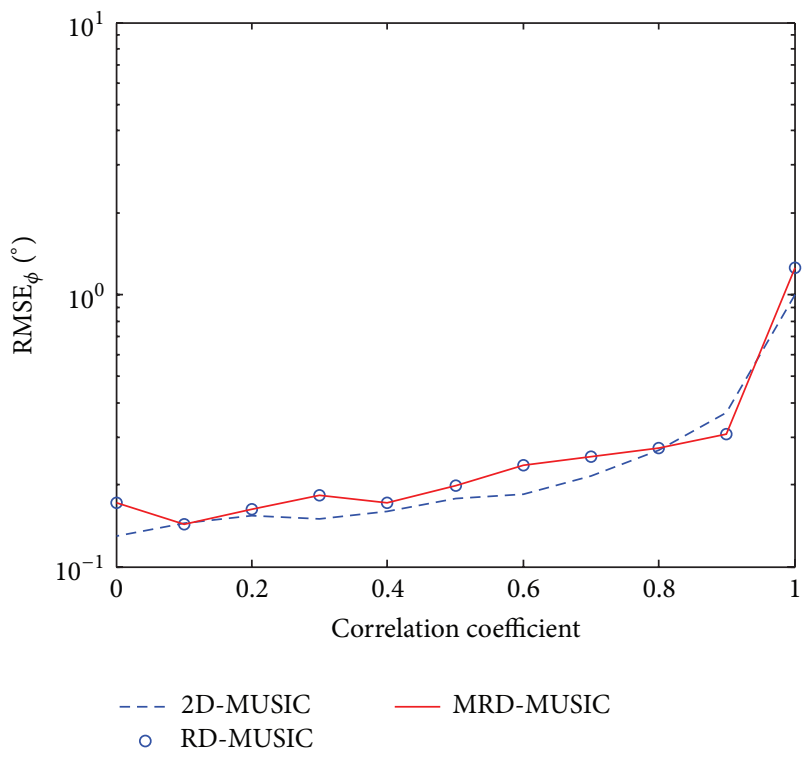

FIGURE 9: RMSE of $\phi$ against correlation coefficient.

\section{Conclusion}

We have proposed a novel efficient NSRD-MUSIC algorithm in this paper for 2D direction-of-arrivals (DOAs) estimation. The new method exploits the specific geometries of URAs to estimate 2D DOAs with efficient 1D spectral search, which costs a significantly reduced computational complexity as compared to 2D-MUSIC. Simulations indicate that NSRDMUSIC which requires no pair matching has a significantly improved accuracy as compared to ESPRIT.

\section{Conflict of Interests}

The authors declare that there is no conflict of interests regarding the publication of this paper. 


\section{Acknowledgments}

This work is supported by National Natural Science Foundation of China (61501142), Shandong Provincial Natural Science Foundation (ZR2014FQ003), China Postdoctoral Science Foundation (2015M571414), and the Fundamental Research Funds for the Central Universities (HIT.NSRIF.2016102).

\section{References}

[1] H. Krim and M. Viberg, "Two decades of array signal processing research: the parametric approach," IEEE Signal Processing Magazine, vol. 13, no. 4, pp. 67-94, 1996.

[2] A. Khabbazibasmenj, A. Hassanien, S. A. Vorobyov, and M. W. Morency, "Efficient transmit beamspace design for search-free based DOA estimation in MIMO radar," IEEE Transactions on Signal Processing, vol. 62, no. 6, pp. 1490-1500, 2014.

[3] R. O. Schmidt, "Multiple emitter location and signal parameter estimation," IEEE Transactions on Antennas and Propagation, vol. 34, no. 3, pp. 276-280, 1986.

[4] F.-G. Yan, M. Jin, S. Liu, and X.-L. Qiao, "Real-valued MUSIC for efficient direction estimation with arbitrary array geometries," IEEE Transactions on Signal Processing, vol. 62, no. 6, pp. 1548-1560, 2014.

[5] F. Yan, M. Jin, and X. Qiao, "Low-complexity DOA estimation based on compressed MUSIC and its performance analysis," IEEE Transactions on Signal Processing, vol. 61, no. 8, pp. 19151930, 2013.

[6] A. Paulraj, R. Roy, and T. Kailath, "A subspace rotation approach to signal parameter estimation," IEEE Transactions on Signal Processing, vol. 74, pp. 1044-1046, 1986.

[7] B. R. Karthikeyan, G. R. Kadambi, and Y. A. Vershinin, "A formulation of 1-D search technique for 2-D DOA estimation using orthogonally polarized components of linear array," IEEE Antennas and Wireless Propagation Letters, vol. 14, pp. 1117-1120, 2015.

[8] G. Wang, J. Xin, N. Zheng, and A. Sano, "Computationally efficient subspace-based method for two-dimensional direction estimation with L-shaped array," IEEE Transactions on Signal Processing, vol. 59, no. 7, pp. 3197-3212, 2011.

[9] F.-J. Chen, C. C. Fung, C.-W. Kok, and S. Kwong, "Estimation of two-dimensional frequencies using modified matrix pencil method," IEEE Transactions on Signal Processing, vol. 55, no. 2, pp. 718-724, 2007.

[10] N. Xi and L. Liping, "A computationally efficient subspace algorithm for 2-D DOA estimation with L-shaped array," IEEE Signal Processing Letters, vol. 21, no. 8, pp. 971-974, 2014.

[11] J.-F. Gu, W.-P. Zhu, and M. N. Swamy, "Joint 2-D DOA estimation via sparse L-shaped array," IEEE Transactions on Signal Processing, vol. 63, no. 5, pp. 1171-1182, 2015.

[12] B. Wu and H. Chen, "DOA estimation and self-calibration algorithm for Y-shaped array in the presence of mutual coupling," in Proceedings of the 2nd International Congress on Image and Signal Processing (CISP '09), pp. 1-5, Tianjin, China, October 2009.

[13] Z. Ye, Y. Zhang, and X. Xu, "Two-dimensional direction of arrival estimation in the presence of uncorrelated and coherent signals," IET Signal Processing, vol. 3, no. 5, article 416, 2009.

[14] M. Rubsamen and A. B. Gershman, "Direction-of-arrival estimation for nonuniform sensor arrays: from manifold separation to Fourier domain MUSIC methods," IEEE Transactions on Signal Processing, vol. 57, no. 2, pp. 588-599, 2009.

[15] Y.-Y. Dong, C.-X. Dong, Z.-B. Shen, and G.-Q. Zhao, "Conjugate augmented spatial temporal technique for 2-D DOA estimation with L-shaped array," IEEE Antennas and Wireless Propagation Letters, vol. 14, pp. 1622-1625, 2015.

[16] A. Swindlehurst and T. Kailath, "Azimuth/elevation direction finding using regular array geometries," IEEE Transactions on Aerospace and Electronic Systems, vol. 29, no. 1, pp. 145-156, 1993.

[17] M. D. Zoltowski, M. Haardt, and C. P. Mathews, "Closed-form 2-D angle estimation with rectangular arrays in element space or beamspace via unitary ESPRIT," IEEE Transactions on Signal Processing, vol. 44, no. 2, pp. 316-328, 1996.

[18] Y.-Y. Wang, L.-C. Lee, S.-J. Yang, and J.-T. Chen, "A tree structure one-dimensional based algorithm for estimating the two-dimensional direction of arrivals and its performance analysis," IEEE Transactions on Antennas and Propagation, vol. 56, no. 1, pp. 178-188, 2008.

[19] J. He, M. N. S. Swamy, and M. O. Ahmad, "Joint DOD and DOA estimation for MIMO array with velocity receive sensors," IEEE Signal Processing Letters, vol. 18, no. 7, pp. 399-402, 2011.

[20] H. van Treesn, Detection, Estimation, and Modulation TheoryPart IV Optimum Array Process, Wiley, New York, NY, USA, 2002.

[21] G. H. Golub and C. H. Van Loan, Matirx Computations, Johns Hopkins University Press, Baltimore, Md, USA, 1996.

[22] F. Li, H. Liu, and R. J. Vaccaro, "Performance analysis for DOA estimation algorithms: unification, simplification, and observations," IEEE Transactions on Aerospace and Electronic Systems, vol. 29, no. 4, pp. 1170-1184, 1993. 

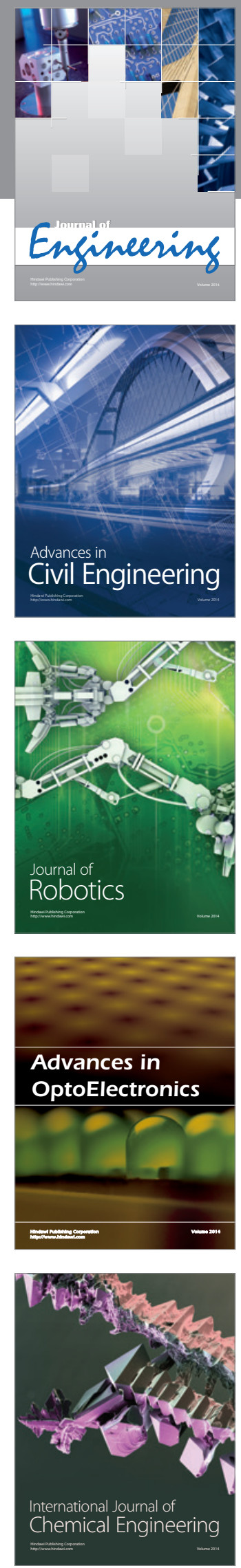

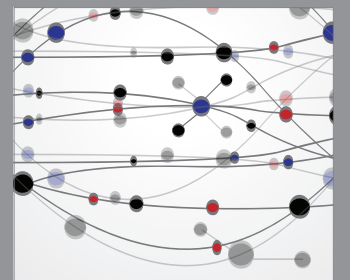

The Scientific World Journal
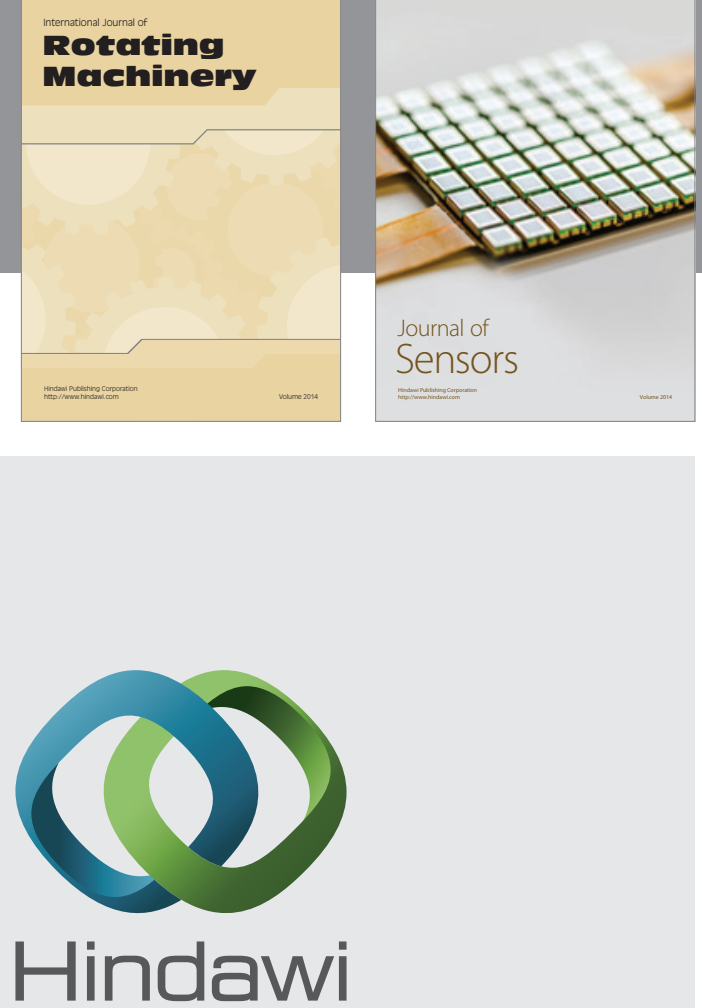

Submit your manuscripts at http://www.hindawi.com
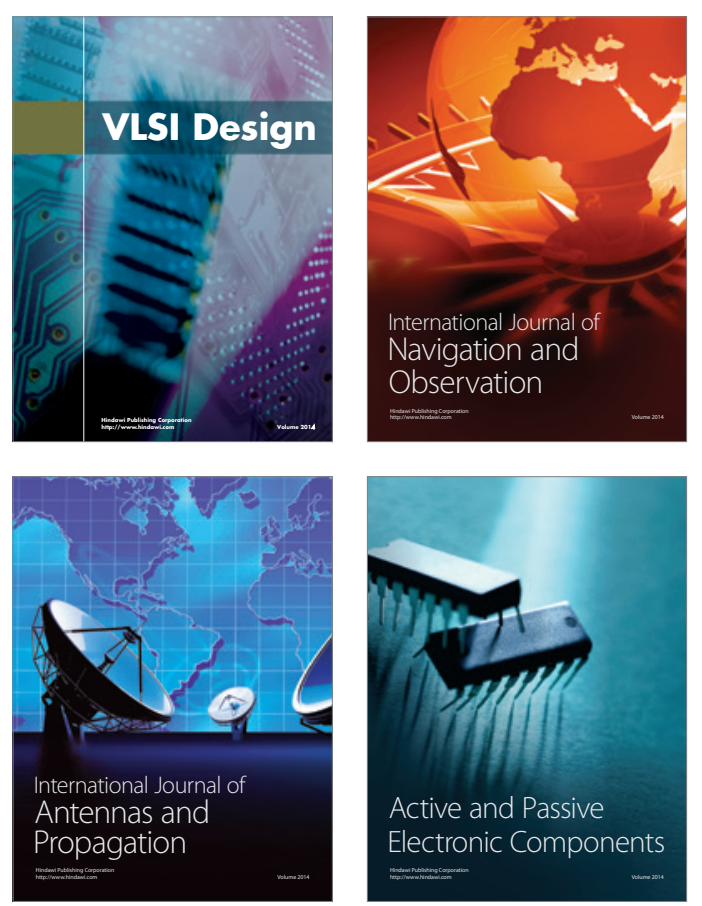
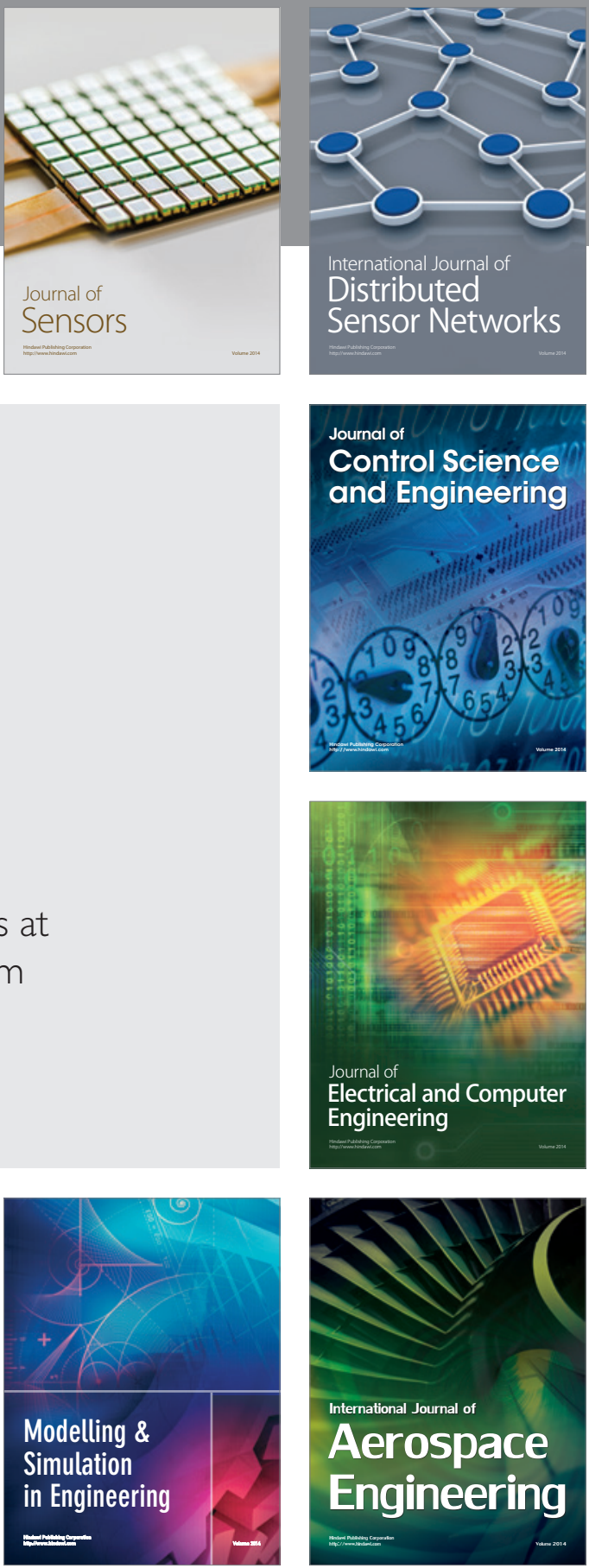

Journal of

Control Science

and Engineering
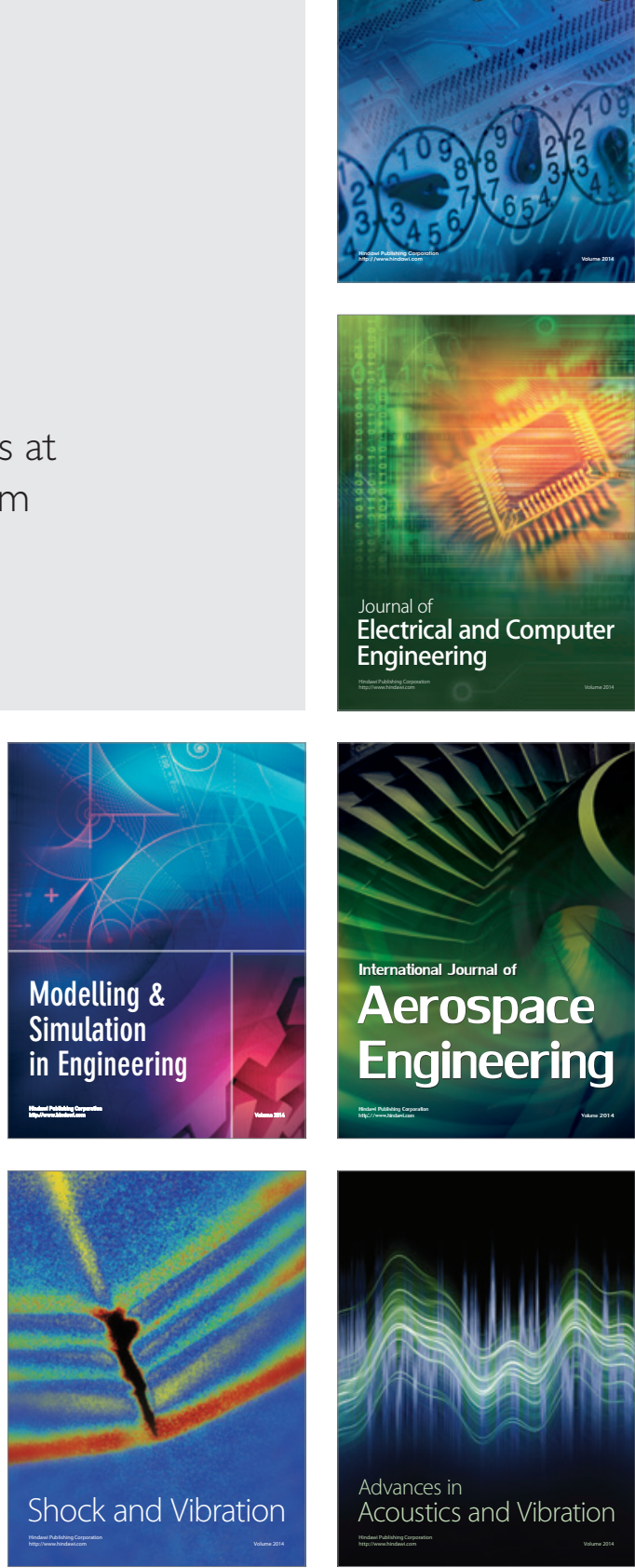